\title{
Laser Beam-Profile Monitor Development at BNL for SNS ${ }^{1}$
}

\author{
R. Connolly, P. Cameron, J. Cupolo, D. Gassner, M. Grau, \\ M. Kesselman, S. Peng and R. Sikora \\ Brookhaven National Lab \\ Upton, NY, USA
}

\begin{abstract}
A beam profile monitor for $\mathrm{H}^{*}$ beams based on laser photoneutralization is being developed at Brookhaven National Laboratory (BNL) for use on the Spallation Neutron Source (SNS) [1]. An $\mathrm{H}^{-}$ion has a first ionization potential of $0.75 \mathrm{eV}$ and can be neutralized by light from a Nd:YAG laser $(\lambda=1064 \mathrm{~nm})$. To measure beam profiles, a narrow laser beam is passed through the ion beam neutralizing a portion of the $\mathrm{H}^{-}$beam struck by the laser. The laser trajectory is stepped across the ion beam. At each laser position, the reduction of the beam current caused by the laser is measured. A proof-of-principle experiment was done earlier at $750 \mathrm{keV}$. This paper reports on measurements made on $200 \mathrm{MeV}$ beam at BNL and with a compact scanner prototype at Lawrence Berkeley National Lab on beam from the SNS RFQ.
\end{abstract}

\section{INTRODUCTION}

Photoneutralization of $\mathrm{H}$ - beams $[2,3,4]$ has been used for measuring beam parameters and for beam manipulation. The first ionization potential of an $\mathrm{H}-$ ion is $0.75 \mathrm{eV}$ which is the energy of a $1.67 \mu \mathrm{m}$ photon. As shown in fig. 1 , any photon with $\lambda<1.6 \mu \mathrm{m}$ can neutralize an $\mathrm{H}$ - ion. In these applications light from a laser is used to mark a portion of the beam. Downstream from the laser interaction point the beam has three components: $\mathrm{H}$ - ions, neutral atoms and unbound electrons. A magnetic field is used to separate one or two of these from the rest of the beam and measurements are made on the remaining beam.

Laser marking of the beam has been done in three ways. The first is to use very short light pulses to neutralize a small phase slice of the entire cross section of the beam. This technique was developed at Los Alamos National Lab to measure longitudinal emittance [5]. Light from a Q-switched Nd:YAG laser was passed through a pulse slicer and frequency doubler to produce 23ps-long pulses. These short light pulses passed through the $\mathrm{H}$ - beam. The charged beam was deflected into a beam stop and a time-of-flight measurement was made on the neutralized beam component to measure momentum spread. A clever modification on this idea using a mode-locked laser and spectrometer was proposed but never built [6].

A second marking technique is to neutralize the entire cross section of the beam with a laser pulse several rf periods long. At Los Alamos this was done to measure the transverse emittance of beams at the exit of an rf cavity [7]. The beam power was

\footnotetext{
${ }^{1}$ SNS is managed by UT-Battelle, LLC, under contract DE-AC05-00OR22725 for the U.S. Department of Energy. SNS is a partmership of six national laboratories: Argonne, Brookhaven, Jefferson, Lawrence Berkeley, Los Alamos, and Oak Ridge.
} 
too great to intercept the full beam with a slit but allowing the beam to drift would introduce space-charge emittance growth. A laser neutralized the full cross section of the beam at the exit of the cavity and then a magnet removed the charged beam. A slit and parallel-channel collector was placed after the clearing magnet. Since the measured beam was neutralized at the cavity exit the actual phase space there could be determined by transforming the measured phase space through a simple drift with no space-charge corrections.

At Fermilab a laser has been used to place a notch in the beam when it sweeps over a Lamberston magnet to reduce activation of the magnet [8]. In this case the neutralized beam hits a beam stop and the charged beam is bent $90^{\circ}$ down the transport line. A $5 \mathrm{~ns}, 99 \%$ notch was produced.

The third marking technique is to focus the laser light into a narrow ribbon and neutralize a small transverse slice of the beam. The transverse profile can be measured by translating the laser 'wire' across the beam and, at each position, measuring the size of the effect it makes on the beam. A measurement which would collect the removed electrons was proposed by D.R. Swenson et al [9]. This paper reports on efforts to develop a laser profile monitor (LPM) at Brookhaven National Lab which measures the beam current notch created by the laser pulse [10].

Profiles of the SNS H' beam will be measured in the medium energy transport line (MEBT) between the radio frequency quadrupole ( $\mathrm{rfq}$ ) and the linac entrance, along the linac, and in the linac-ring transport line. Stepped carbon-wire scanners are the primary profile diagnostic. However beam heating will limit wire scanners to tuning and matching applications with either the beam pulses shortened or the current reduced. Also there are concerns about placing wires near the superconducting cavities where wire failure can cause cavity damage. These concerns have motivated the effort to develop a laser profile monitor (LPM) which is noninvasive.

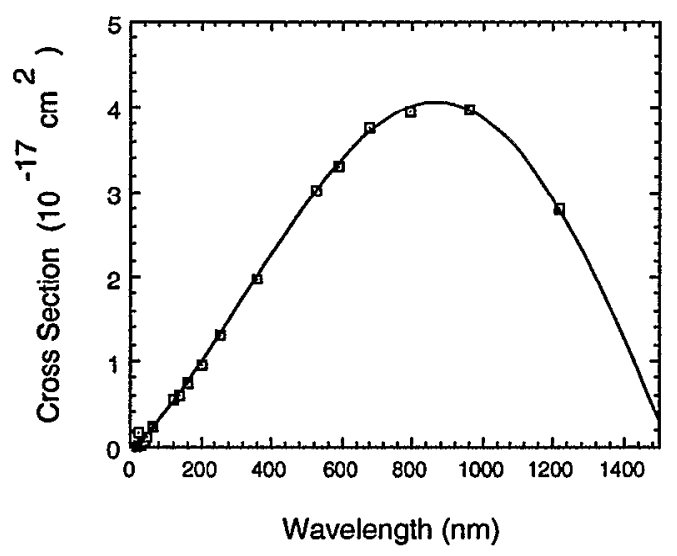

FIGURE 1. Calculated cross section for $\mathrm{H}^{-}$photoneutralization as a function of photon wavelength. Data are from a table in ref. [2].

\section{PHOTONEUTRALIZATION}

Figure 1 shows the photoneutralization cross section as a function of photon wavelength in the center-of-mass frame. If the laser beam crosses the $\mathrm{H}$ - beam at a lab 
angle of $\theta_{\mathrm{L}}$ the photon energy in the moving frame is Lorentz shifted by the amount $[11,12]$,

$$
\mathrm{E}_{\mathrm{CM}}=\gamma \mathrm{E}_{\mathrm{L}}\left[1-\beta \cos \left(\theta_{\mathrm{L}}\right)\right]
$$

For the SNS laser installation $\theta_{\mathrm{L}}$ will be $90^{\circ}$ so at the full energy of $1 \mathrm{GeV}$ the centerof-mass photon energy will be double the lab energy. For these measurements and probably for the final SNS installation the laser will be a Q-switched Nd:YAG laser operating at its fundamental of $\lambda=1064 \mathrm{~nm}$ so at the full energy of $1 \mathrm{GeV}$ the neutralization cross section will be about $70 \%$ of the low encrgy cross section. is,

The fraction of beam ions which get neutralized passing through the laser beam

$$
f_{\text {neut }}=1-\mathrm{e}^{-\sigma(\mathrm{E}) \mathrm{Ft}}
$$

Here $\sigma(\mathrm{E})$ is the energy-dependent cross section, $\mathrm{F}$ is the photon flux, and $t$ is the time the ion is in the light beam. The photon flux in the moving reference is also transformed the same as the photon energy [13],

$$
\mathrm{F}_{\mathrm{CM}}=\gamma \mathrm{F}_{\mathrm{L}}\left[1-\beta \cos \left(\theta_{\mathrm{L}}\right)\right]
$$

The neutralization fraction from a given laser initially drops with increasing beam energy then becomes almost flat from $400 \mathrm{MeV}$ to $1 \mathrm{GeV}$. In this range the decreased reaction cross section from the Lorentz boosted photon energy is approximately offset by the Lorentz boost in photon flux.

For example, the laser on the SNS MEBT experiment produces a 20ns-long pulse with an output energy of $50 \mathrm{~mJ}$. It is focused to a rectangular spot $1 \mathrm{~mm}$ wide by $3 \mathrm{~mm}$ along the beam. The approximate variation of neutralization fraction with beam energy this laser will produce is shown in fig. 2. This calculation does not include the Lorentz shift of neutralization cross section. Higher power lasers are required for higher beam energies to achieve the same signal level in the detector.

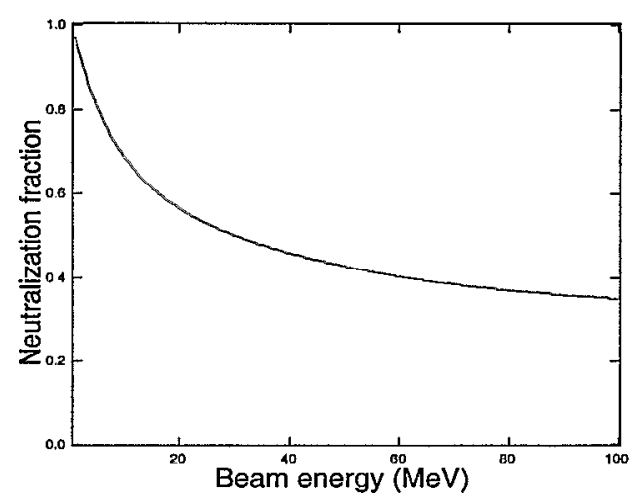

FIGURE 2. Calculated neutralization fraction vs. beam energy for a $20 \mathrm{~ns}-1 \mathrm{ong}, 50 \mathrm{~mJ}$ laser pulse focused to a spot size of $1 \mathrm{~mm} \times 3 \mathrm{~mm}$. 


\section{THE $750 \mathrm{KeV}$ EXPERIMENT}

Our first profile measurement was made on the BNL linac between the rfq and the first drift tube linac tank, fig. 3. A light pulse from a Q-switched Nd:YAG passed through the $750 \mathrm{keV} \mathrm{H}^{-}$beam from the linac rfq neutralizing most of the beam the light passed through. A downstream current transformer measured a dip in the beam current which was proportional to the fraction of the beam hit with the light, fig. 4 . The laser beam was stepped across the ion beam and the profile constructed by plotting the depth of the current notch vs. laser beam position.

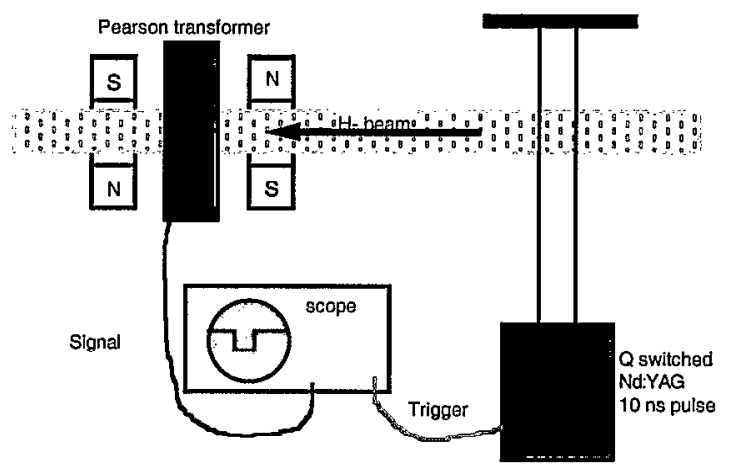

FIGURE 3. Laser scanner experiment on BNL linac. The first of two $10 \mathrm{Gm}$ dipole magnets removes the free electrons from the beam and the second straightens the beam.

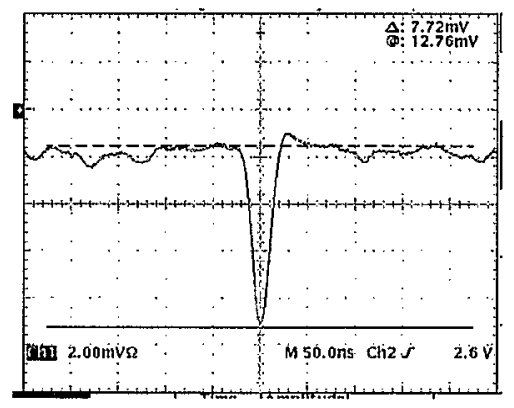

FIGURE 4. Scope trace of the current transformer signal showing notch created by the laser pulse.

The arrangement of the laser and optics on the linac beamline is shown in fig. 5. A CFR200 laser from Big Sky Laser [14] was mounted on a shelf at the top left. Three $45^{\circ}$ mirrors were mounted inside the vacuum on linear motion feedthroughs. The topleft mirror was used to switch between vertical and horizontal scans and the other two did the scanning. The top-right mirror scanned horizontally and the bottom-left mirror scanned vertically. Both scanning mirrors are shown with arms to hold lenses. In this experiment the lenses were not installed.

The CFR200 puts out $200 \mathrm{~mJ}$ pulses that are about $20 \mathrm{~ns}$ long. Without lenses the beam diameter is about $0.6 \mathrm{~cm}$ giving a photon flux of $1.9 \times 10^{26} / \mathrm{cm}^{2} \mathrm{~s}$. About $97 \%$ of 
the ions passing through the center of the laser beam were neutralized. The laser is triggered $400 \mu \mathrm{s}$ before the measurement is to made. The laser then returns a timing pulse synchronous with the Qswitch firing which was used to trigger a scope.

When an ion is neutralized the free electron continues to move along with the beam. These electrons have to be removed from the beam to measure a current drop. In an accelerator installation this is accomplished by either rf cavities or quadrupoles but in the experiment the current transformer had to be placed in the same vacuum chamber as the laser optics. For this reason we placed two weak permanent-magnet dipoles on either side of the transformer. The pole tips are $2.5 \mathrm{~cm}$ square and $5 \mathrm{~cm}$ apart and the field is about $400 \mathrm{G}$. The first magnet deflects the electrons from the beam and the second one straightens out the beam.

The data were taken by moving the mirrors manually and measuring the notch depth on an oscilloscope set to average 15 shots. We measured a maximum notch depth of about $40 \%$ on the horizontal scan. If the laser beam power was uniformly distributed over the spot the maximum notch depth should have been closer to $60 \%$. Based on this we conclude the laser power was not uniform over the spot.

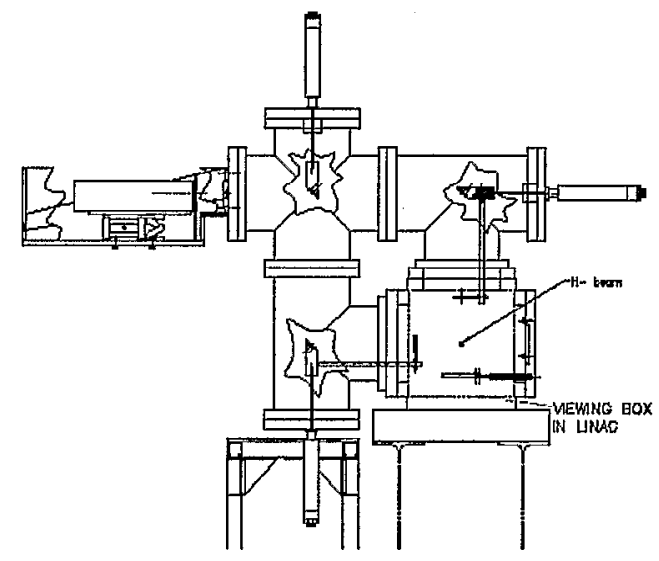

FIGURE 5. Laser scanning assembly installed on linac beamline. View is looking up beamline.

\section{Measured Profiles}

Figure 6 shows the measured horizontal and vertical profiles. In each plot the measured points are indicated by markers and the curve is a gaussian fit to the data.
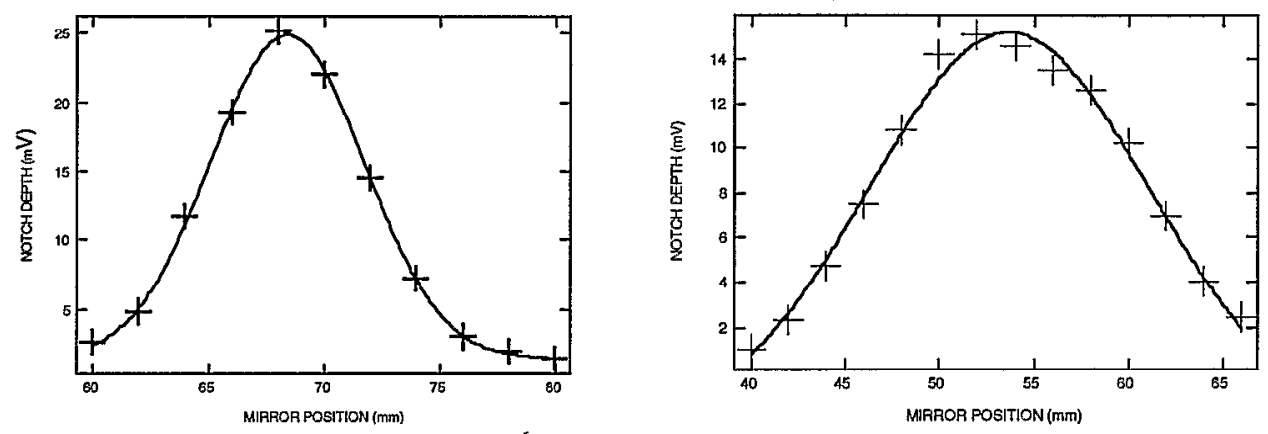

FIGURE 6. Measured horizontal (left) and vertical beam profiles. 
The rms widths of the two fitted curves are $\sigma_{x}=3.32 \pm 0.05 \mathrm{~mm}$ and $\sigma_{y}=7.3 \pm 0.6$ $\mathrm{mm}$. These values agree with expectations from previous measurements at this location, however for this experiment there was no profile measurement by another method. We had a carbon wire installed in the beam box but we were operating parasitically during a production run and we were never able to get the short beam pulses necessary to prevent damage to the wire.

\section{THE MEBT EXPERIMENT, $2.5 \mathrm{MeV}$}

Based on the promise of the BNL linac experiment we designed a laser platform which would attach to one of the wire-scanner chambers on the SNS MEBT at Lawrence Berkeley National Lab. Mounted on the platform, fig. 7, are a $50 \mathrm{~mJ} / \mathrm{pulse}$ laser head, a lens holder, and three linear actuators which move $45^{\circ}$ mirrors in a more compact but otherwise identical arrangement to the linac experiment of fig. 5. There are four profile measurement stations. Each has a wire scanner and four windows for laser-beam access. We installed the platform on the most upstream chamber after the MEBT was under vacuum and ready for first beam.

A $300 \mathrm{~mm}$-focal-length cylindrical lens is mounted directly in front of the laser head and the two optical path lengths from the lens to the beam center are the same. The lens produces a $1 \mathrm{~mm}$ wide by $3 \mathrm{~mm}$ long light ribbon across the beam producing a measurement window of $\mathrm{rms}$ width $0.3 \mathrm{~mm}$. Since the measured rms width of the horizontal profile is $1.48 \mathrm{~mm}$ the width of the laser beam caused about $2 \%$ broadening of the profile.

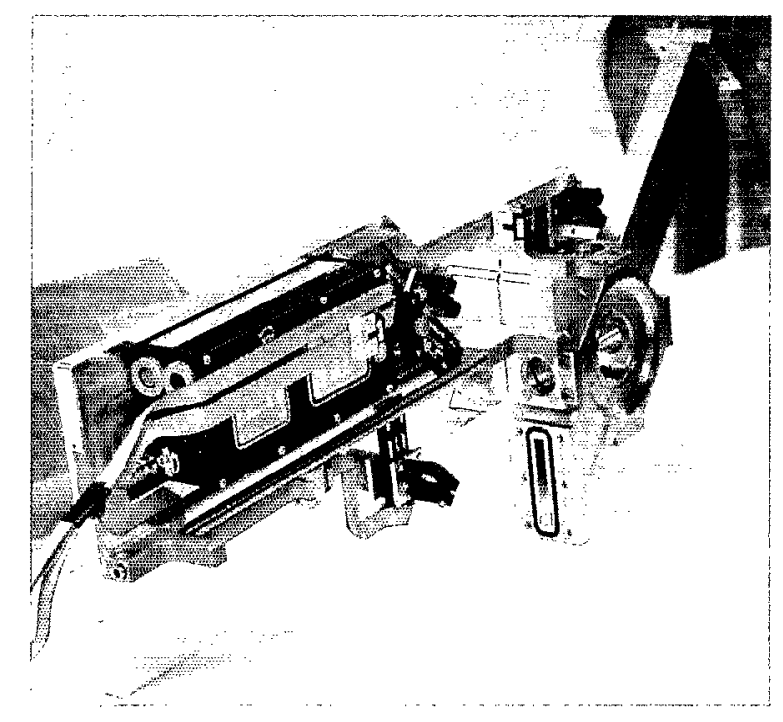

FIGURE 7. Laser scanning platform mounted on the SNS MEBT wire scanner chamber.

The signal was detected with the existing beam transformer at the end of the MEBT. The BCM signal was split and our half was fed into a LeCroy LT374L scope 
with ethernet connection [15]. A math channel on the scope was used to average for several pulses to reduce noise and rf pickup. The rf pickup could have been greatly reduced with the use of a $50 \mathrm{MHz}$ low-pass filter as was done on the $750 \mathrm{keV}$ experiment but none was available. In the profiles shown in fig. 8 , the signals from 25 beam pulses were averaged giving about $40 \mathrm{~dB}$ signal/background in the beam center.

The experiment was controlled in Labview. The program switched the laser, moved the mirrors, initialized the scope for each new position, and read the data. Cursors on the scope were set manually around the pulse. For each set of averaged data the program summed the channels between the cursors, summed an equal number of channels before the pulse, and subtracted these two 'integrals' to give one data point in the profile.
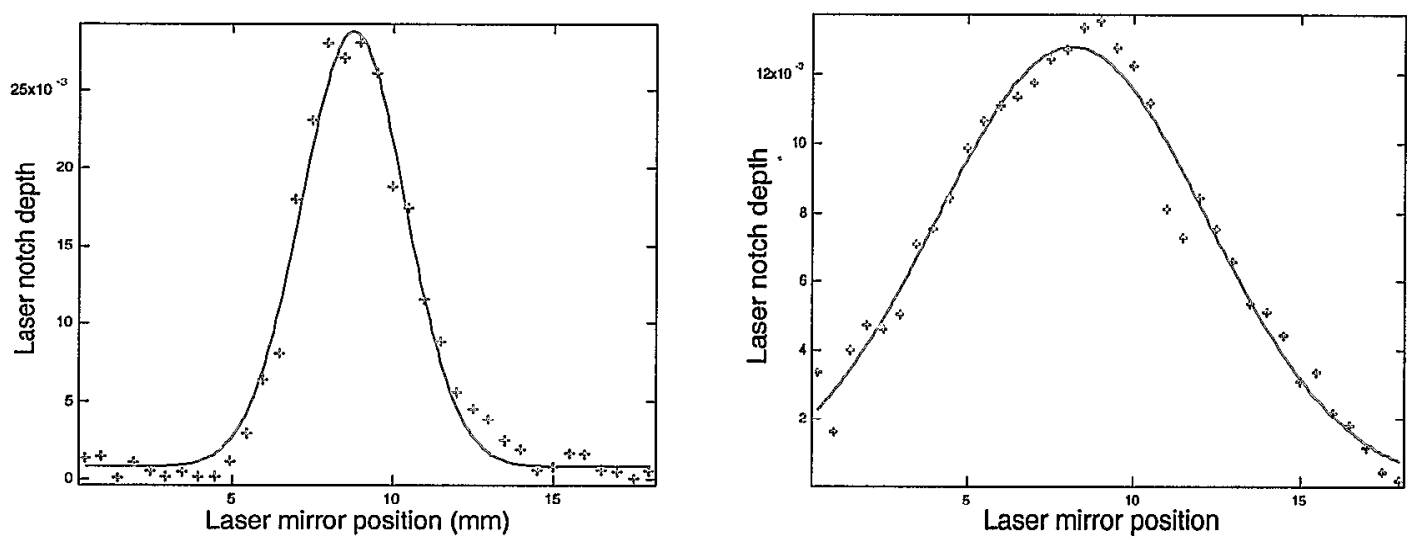

FIGURE 8. Beam profiles measured on the SNS MEBT with the laser profile monitor. The horizontal profile (left) has a measured width of $\sigma=1.60 \pm 0.04 \mathrm{~mm}$ and the vertical profile has $\sigma=4.16 \pm 0.16 \mathrm{~mm}$.

\section{MEASUREMENTS AT $200 \mathrm{MeV}$}

After the measurements on the BNL linac at $750 \mathrm{keV}$ the entire apparatus shown schematically in fig. 5 was moved to the high energy end of the linac to measure $200 \mathrm{MeV}$ beam. It was installed in the linac-AGS transfer line which is no longer used for beam transport, fig. 9 . The $200 \mathrm{~mJ}$ laser hear is mounted on the covered shelf at the top right. The beam line chamber also had the carbon wire installed.

In this installation there were two cylindrical lenses which moved with each mirror. A $300 \mathrm{~mm}$-focal-length lens produced a waist perpendicular to the ion beam and a $50 \mathrm{~mm}$ lens spread the light beam longitudinally to reduce power density on the laser beam stop. The light beam which crossed the ion beam was $1 \mathrm{~mm}$ wide by $20 \mathrm{~mm}$ long. The calculated neutralization of beam passing through the laser light is $72 \%$.

For this experiment the goal was to use stripline beam position monitors (BPMs) to measure the laser notch. A single-plane RHIC BPM was installed before and after the beam box. In the superconducting linac of SNS there are BPMs between rf tanks with a current transformer at the exit of the linac. Using the striplines as detectors gives us access to a upstream and downstream detector spaced by a single if structure. 
Using the transformer for signal detection will require good transmission through the full linac before profiles can be made at any point.

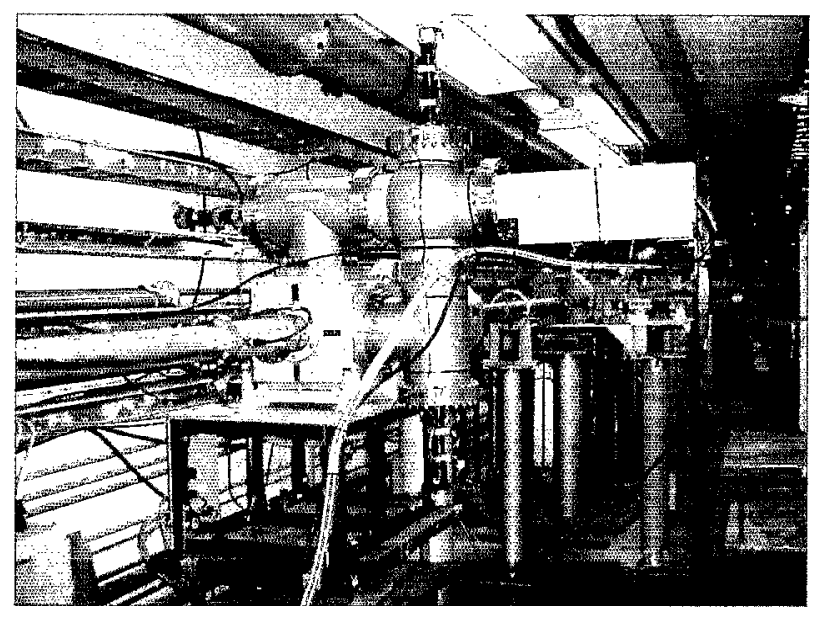

FIGURE 9. LPM measurement station at $200 \mathrm{MeV}$ in the BNL linac.

To measure the notch we adjust the phase and attenuation of two stripline signals and combine them with a hybrid to produce a nulled signal in the absence of laser neutralization. The laser pulse causes a signal imbalance which appears either as a wide or narrow spot in the rf envelope depending on how the signals are combined. Different from the transformer measurements, now the signals are bipolar with no dc component. To integrate the laser notch, the scope data are passed through a Labview VI which takes the absolute value of each data point.

Also different from the first two experiments, we have had extremely noisy beam at $200 \mathrm{MeV}$. However with care we have been able to match the beam pattern from the upstream and downstream BPMs and produce a null signal with over $20 \mathrm{~dB}$ common mode rejection. Figure 10 shows a scope trace of the laser notch in the nulled signal.

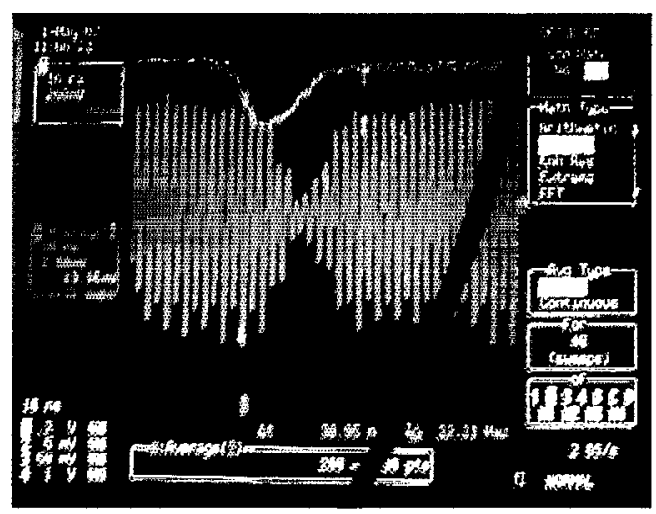

FIGURE 10. Laser notch in the beam rf envelope picked up on the BPM striplines. The top trace is the signal from a photodiode near the laser head. 


\section{Experimental Difficulties}

The first two experiments were conducted at low energy, where we had beamline access with the beam on, and inside clean vacuum systems. At $200 \mathrm{MeV}$ we have had to operate remotely from outside the tunnel. Also we have been in a section of beam line which normally is not used to transport beam so we have had limited beam time.

There was a period of two months between installation of the experiment and available beam during which time the laser controller was in the tunnel. Less than a month after we started getting beam time the laser controller failed from radiation and had to be replaced. The beamline is thirty years old and is slightly contaminated with pump oils. All the optics are in the vacuum and, during the wait for beam, they became contaminated with oil. When we started taking data the laser light burned the oil on the optics forming milky patches which scattered the light. During the early measurements the signal continued to get worse over time. We discovered the damaged optics when replacing the damaged laser.

\section{Profile Measurements}

For several reasons including those mentioned above the measurements at $200 \mathrm{MeV}$ have not been as clean as the low-energy ones. The first profile data at 200 $\mathrm{MeV}$, fig. 11 , made with $100 \mu \mathrm{A}$ of beam from the polarized source, was taken by hand by measuring the notch depth with the scope cursors.

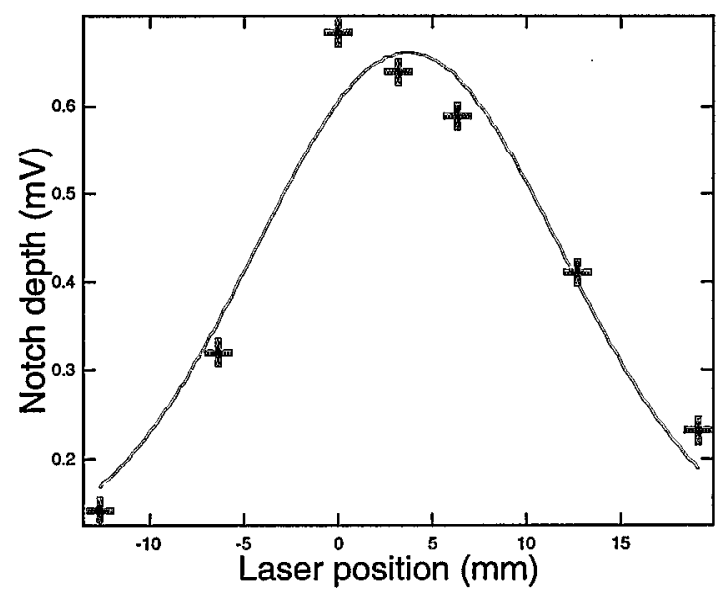

FIGURE 11. Profile of $100 \mu$ A polarized beam. The width of the fitted gaussian is $8.1 \pm 2.2 \mathrm{~mm}$.

After the polarized run we had several weeks of $400 \mu \mathrm{s}-1 \mathrm{ong}, 10 \mathrm{~mA}$ beam pulses before the laser failed from radiation. During the early portion of this time we were able to get clean laser signals but as time progressed the signal progressively got worse. In hindsight we realize this was from the oil contamination problem.

Figure 12 shows a LPM-measured profile and a wire-scanner profile taken the day before the LPM profile. We were unable to get a LPM profile and wire profile at the same time because the wire scanner failed after one use. The linac had been returned to the set points of the previous day although between the two times we had beam the 
linac had been switched back to normal production operation. The measured widths are: $\sigma_{\mathrm{LPM}}=5.3 \pm 1.3 \mathrm{~mm}$ and $\sigma_{\text {wire }}=3.51 \pm 0.34 \mathrm{~mm}$.
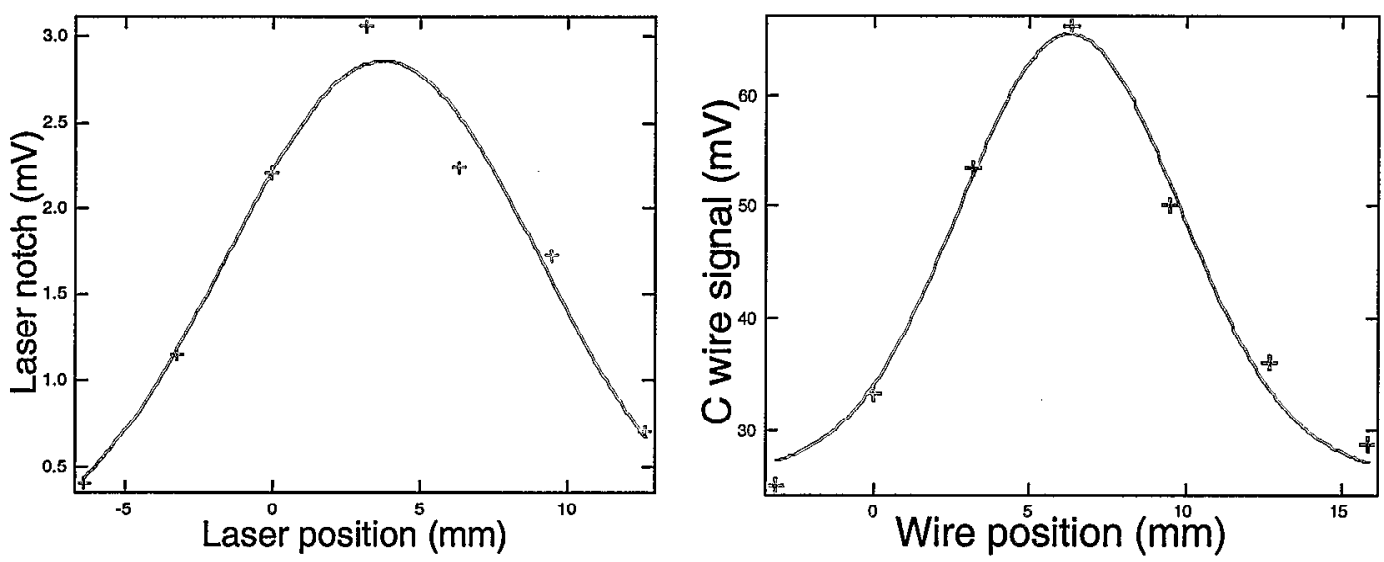

FIGURE 12. Beam profile taken with a wire scanner (right) and a beam profile taken the next day with the laser scanner (left). Profiles were taken on different days but the linac had been restored to the set points of the previous day.

Our last beam run before this paper produced a fully-automatic horizontal profile scan. The Labview software was the same as that used at LBNL with the beam current transformer. Since the stripline data are bipolar with no dc component we added an absolute value VI to rectify the scope data then summed the data between cursors to produce a notch integral. Figure 13 shows the rectified scope trace of the laser notch. Figure 14 shows the laser profile and a profile taken with the carbon wire at the same time. With the laser the width of the fitted gaussian is $\sigma=2.82 \pm 0.76 \mathrm{~mm}$ and the wire measured $\sigma=3.61 \pm 0.22 \mathrm{~mm}$.

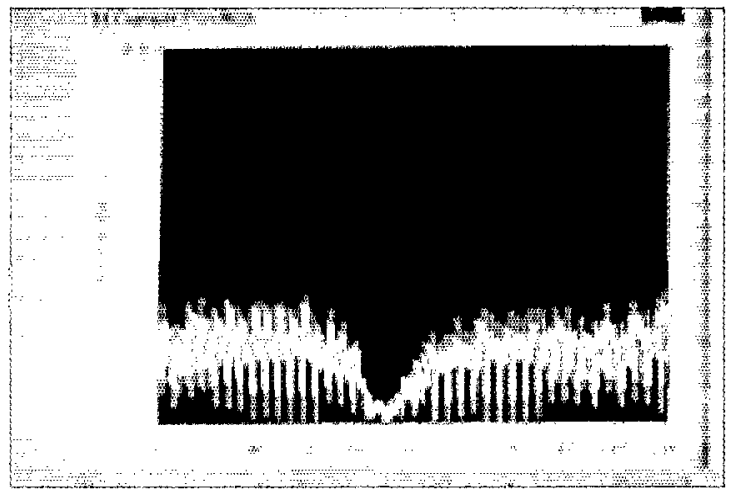

FIGURE 13. At each laser position the scope averaged forty traces. The scope was read into Labview and passed through an absolute value VI to rectify the bipolar signal. Cursors on the scope marked the notch channels and all of the data between the cursors were added to produce an integral. 

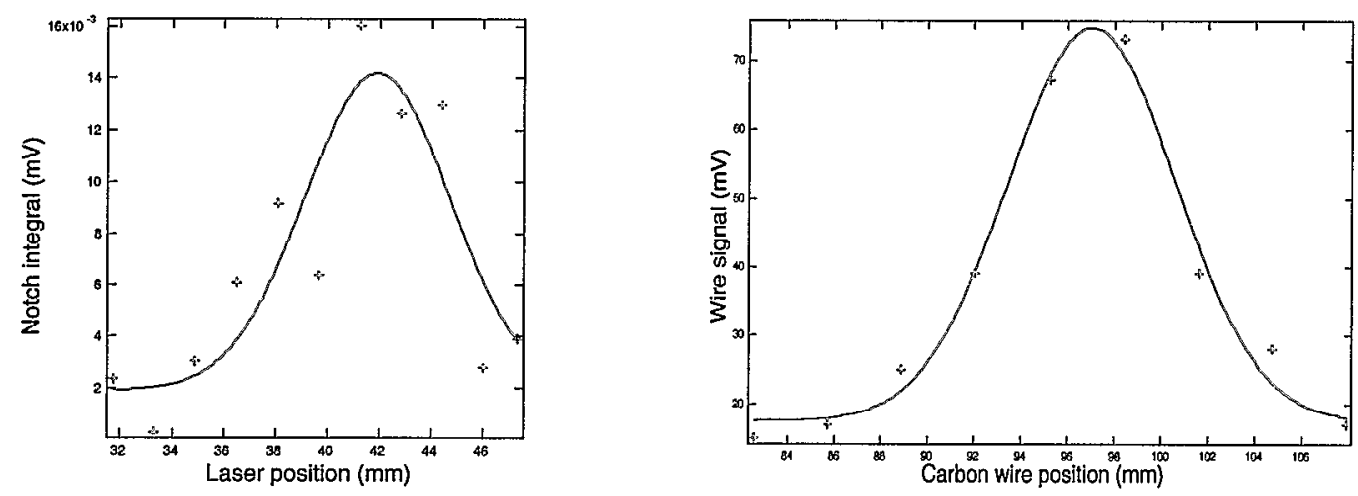

FIGURE 14. The fit to the laser date on the left gives a width of $\sigma=2.82 \pm 0.76 \mathrm{~mm}$. The wire profile on the right measured $\sigma=3.61 \pm 0.22 \mathrm{~mm}$.

\section{DISCUSSION}

Transverse profiles of $\mathrm{H}$ - beams can be measured by scanning a laser beam across the ion beam and detecting the notch in the beam current downstream. This technique is attractive because no components are in the vacuum, profile measurements can be made without disrupting machine operation, and measurements can be made on high power beams. As we demonstrated on the SNS MEBT, profile measurement capability can be added to an operating accelerator if a suitable window exists and a downstream current transducer is available.

Q-switched Nd:YAG lasers are perfect for these measurements on beams with energies up to about $1 \mathrm{GeV}$. These lasers are readily available with a wide range of output energies. Lasers with pulse energies of close to a Joule are available with compact laser heads attached to power units by umbilicals which make them suitable for mounting on compact platforms on beamlines.

Our experiments have placed the laser controller and cooling unit next to the beamline for convenience. Two laser controllers have failed from radiation. Any installation of a LPM in a radiation area has to have the controller in a nonradiation area. We do not know the radiation doses the laser heads can take. The plan for SNS is to place the entire laser outside of the tunnel and transport the light by mirrors or fiber optics.

The two LPM experiments which used beam current transformers for beamcurrent detection produced extremely clean signals with very little set up time. The measurement at $750 \mathrm{keV}$ gave a signal/noise ratio at beam center of $25 \mathrm{~dB}$ and the MEBT experiment gave $40 \mathrm{~dB}$. Measurements on $200 \mathrm{MeV}$ beam with BPM striplines have been made but we need to make improvements in the data processing. 


\section{ACKNOWLEDGEMENTS}

The authors thank Brian Briscoe and Vinnie LoDestro at the BNL linac. Also we thank Alex Ratti, Larry Doolittle and John Staples at LBNL for letting us try the MEBT platform during commissioning. SNS personnel who have become involved include Saheed Asadi, Warren Grice, Sasha Alexander and Tom Shea.

\section{REFERENCES}

[1] http://www.sns.gov/

[2] J.T. Broad and W.P. Reinhardt, Phys. Rev. A14 (6) (1976) 2159.

[3] M.J.Ajmera and K.T. Chung, Phys. Rev. Al2 (2) (1975) 475.

[4] M. Daskhan and A.S. Ghosh, Phys. Rev. A28 (5) (1983) 2767.

[5] W.B. Cottingame, G.P. Boicourt, J.H. Cortez, W.W. Higgins, O.R. Sander and D.P. Sandoval, Proc. 1985 Particle Accelerator Conf., IEEE Trans. Nucl. Sci. NS-32 (1985) 1871.

[6] V.W. Yuan, R.C. Connolly, R.C. Garcia, K.F. Johnson, K. Saadatmand, O.R. Sander, D.P. Sandoval and M.A. Shinas, "Measurement of longitudinal phase space in an accelerated $\mathrm{H}^{-}$beam using a laser-induced neutralized method", Nucl. Instr. and Meth. A329 (1993) 381-392.

[7] R.C. Connolly, K.F. Johnson, D.P. Sandoval and V. Yuan, "A transverse phase-space measurement technique for high-brightness $\mathrm{H}^{-}$beams", Nucl. Instr. and Meth. A132 (1992) 415-419.

[8] R. Tomlin, "Ion Beam Notcher Using a Laser", FERMILAB-Conf-01/117-E (2001).

[9] D.R. Swenson, E.P. MacKerrow and H.C. Bryant, "Non-Invasive Diagnostics for H- Ion Beams using Photodetachment by Focused Laser Beams", Proc. 1993 Beam Instrumentation Workshop (Santa Fe). A.I.P. Conf. Proceedings, (319), 343.

[10] R. Connolly, P. Cameron, J. Cupolo, M. Grau, M. Kesselman, C-J Liaw and R. Sikora, "Laser Profile Measurements of an H- Beam", Proc. 5th European Workshop on Beam Daignostics and Instrumentation, (Grenoble), http:/www.esrf.fr/conferences/DIPAC/DIPAC2001Proceedings.html

[11] R.E. Shafer, "Laser Diagnostic for High Current H- Beams", Proc. 1998 Beam Instrumentation Workshop (Stanford). A.I.P. Conf. Proceedings, (451), 191.

[12] R. Tomlin, "Laser Stipping of Relativistic H- Ions with Practical Considerations", FERMILABTM-1957, (1995).

[13] S.M. Shafroth and J.C. Austin, Eds., Accelerator-Based Atomic Physics Techniques and Applications, A.I.P. Press (1997) Chap. 6.

[14] Big Sky Laser Technologies, Inc., Bozeman, MT 59715.

[15] LeCroy Corp., Chestnut Ridge, NY 10977. 